\begin{tabular}{ccc}
\hline & International Journal of Engineering \& Technology, 7 (2.8) (2018) 688-691 \\
SPC & International Journal of Engineering \& Technology \\
Website: $w w w . s c i e n c e p u b c o . c o m / i n d e x . p h p / I J E T$ & Research Paper \\
\hline
\end{tabular}

\title{
Detection of brain tumour using segmentation
}

\author{
V.V.Prakash ${ }^{1 *}$, K.Manjunathachari ${ }^{2}$ \\ ${ }^{1}$ Research Scholar, ${ }^{2}$ Professor \\ ${ }^{1,2}$ Department of Electronics and Communication Engineering, GITAM University, Hyderabad \\ *Corresponding Author Email: prakash.vv@gmail.com
}

\begin{abstract}
This paper presents the detection of the brain tumour by improvised image segmentation technique. The Magnetic Resonance Image is considered for the purpose of detecting the brain tumour. The human brain occupies $2 \%$ of the body's weight. The Magnetic Resonance Image is preferred over the CT scan image for accuracy of the MRI based brain tumour detection is high. Though there are several techniques of segmentations, the water shed proves to be advantages in detecting the region of interest. The score plot of the vertical and horizontal plane exhibits high efficiency and feasibility of the technique presented.
\end{abstract}

Keywords: Human Brain Tumour, Magnetic Resonance Image, Water Shed Segmentation.

\section{Introduction}

The brain is the central processing unit of any living being. The human brain consists of the billions of neurons referred as Gray Matter and billions of fibrous nerves referred as White Matter. Depending on the origin, the brain tumour might be classified into two types. They are a) Benign tumour and b) Malignant tumour. Benign tumour is developed from the abnormal behaviour of the brain cells. Malignant tumour is developed in other organs and spread into the brain. The malignant tumour is also referred as cancerous tumour.

Externally, the causes for brain tumor are exposure to vinyl chloride and ionizing radiations. Also, there are some inherited diseases which have different grades of tumours based on the severity level. In human brain, the nature of substances permissible inside the brain is controlled by the Blood Brain Barrier (BBB). If any tumour is to be developed in the brain, the BBB has to be broken. This BBB disruption of the BBB is well captured by using the MRI or CT image. The MRI scan is often used for scanning the benign brain tumours which indicates the tumour darker than brain tissues. The non-invasive MRI based brain tumour imaging technique is attractive and attentive in recent years [1].

There are several techniques used in image for the detection of tumour in human brain. The brain tumour identification technique using the intelligent water drops has detection accuracy increase by $20 \%$ and delay is reduced by 1.5 seconds [2]. The histogram differencing based approach is applied to segment the tumour pixels in the MRI scan [3]. The soft computing based image segmentation plays an important role in detection of tumour detections. The fuzzy logic based brain tumour from the MRI scan avoids misclustering of regions in the image by using morphological filter[4][5].The neural network based brain tumour identification techniques yields better sensitivity, specificity and accuracy compared to the conventional method [6]. Berkeley wavelet transformation (BWT) based brain tumour segmentation shows accuracy of $96.51 \%$, specificity of $94.2 \%$ and sensitivity of $97.72 \%$.[7].The Gaussian mixture model for the Glioblastoma feature extraction using the T1-WI and T2-WI depict the accuracy performance of $97.05 \%$ [8].

Nowadays fusion of two different technologies is used for collective advantages. The MRI and CT images are combined together could easily detect the tumour cells using the discrete wavelet transform [9]. The fusion of threshold segmentation and morphological extraction gives a perfect picture of the tumour structure [10].

In this paper, the water shed algorithm is utilized for the purpose of detecting the brain tumour using the MRI scan. The water shed algorithm for the detection of brain anomalous regions proves to be highly enhanced and accurate [11]. Controllers analyses for non-linear systems has been reported [12-21].

\section{The Proposed Method}

The proposed methodology involves in the conversion of MRI colour image to Gray scale Digital image. The GDI equivalent is detected for brain tumour in the image through the processes of filtering, marking and identifications. The final process of segmentation is performed for the brain tumour image. The design flow for the proposed method is given in Fig.1.

\section{A. MRI Image}

Magnetic Resonance Image (MRI) is utilized in the field of medical imaging. The basic concept behind the MRI scanning is the excitation of magnetic field over the hydrogen atom of the living organisms. Naturally, hydrogen atom is present in the form of water and fat content in the living organisms. For this reason, the MRI scans the water and fat in the area of interest. In this work, the MRI image of the human skull is considered. The human skull neuron image obtained is a T2-weighed image. Based on the type of organs scanned; the MRI could be used for medical applications.

\section{B. Pre-processing}

The MRI has to be converted into digital format for performing manipulation. The MRI is converted to the Grey scale Digital 
Image (GDI) for processing. The GDI is also referred as black and white image composed with shades of gray. This GDI is useful in the measurement of the intensity of each pixel in a single band of the electromagnetic spectrum. The resolution of the GDI is $2^{8}$ bits. The intensity scale for each pixel ranges from 0 to 255 values, where the value ' 0 ' stands for black and ' 255 ' stands for white. The corresponding binary equivalents are " 00000000 " for black and "11111111" for white. The MRI brain skull image is converted to the GDI for pre-processing.

\section{Edge detection}

Edge detection is the mathematical process performed on the GDI image pixels to identify the occurrence of the drastic intensity changes in the neighbouring pixels. The edge detection is a continuous iteration method that evaluates the discontinuous in the brightness of all pixels in the GDI converted image.

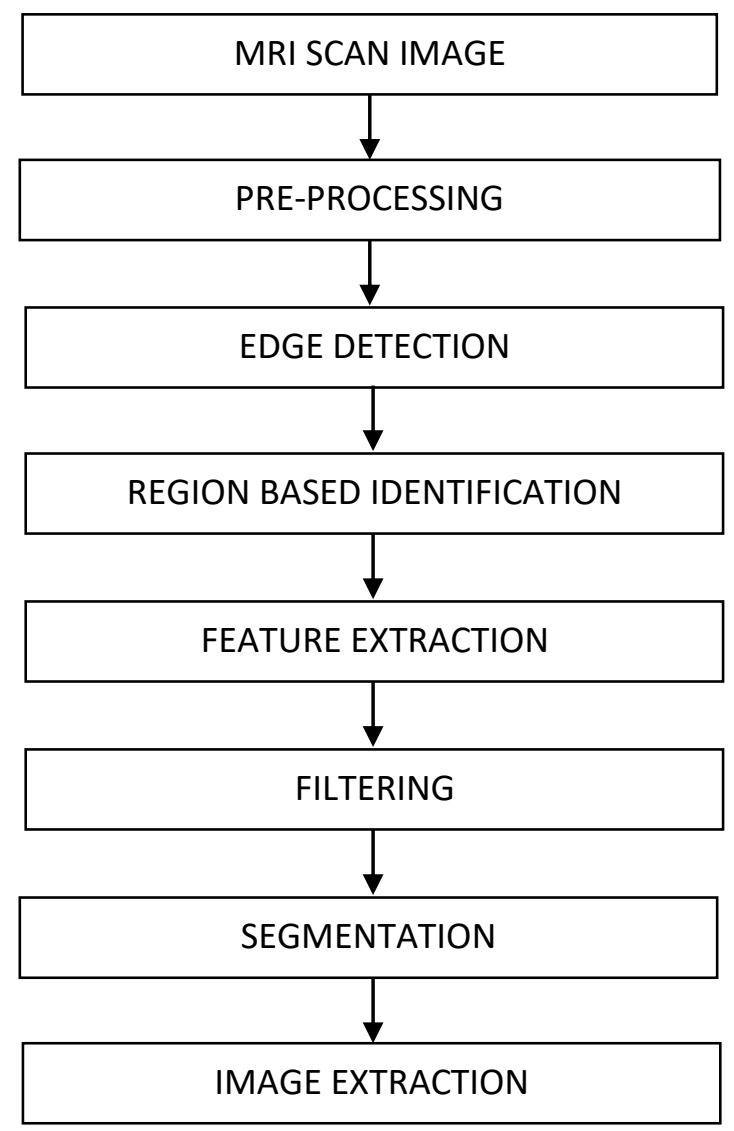

Fig. 1: Design flow for the brain tumour identification using water shed segmentation

\section{Region based Identification}

The edge detection of GDI helps in the determination of the boundaries of the tumour in the brain. The desired region of tumour or object is referred as the Region of Interest (ROI).

\section{E. Feature extraction}

The process of feature extraction is to reduce the number of pixels for particular operations. The isolation of desired portion of the image is obtained by the feature extraction algorithms. After the desired portion is identified, it is marked by the different colour.

\section{F. Filtering}

Filtering is the processing of eradicating unnecessary noise pixels in the image. The use of high pass filter improves the quality of image and reduces manipulation errors. The impulse noise of the image is subjected to another filtering technique called as "Median filter". The median filter utilizes the statistical median calculation of each pixel by considering the surrounding neighbouring pixels.

\section{G. Segmentation}

Though segmentation is performed by several ways, the intensity based segmentation is prominent in operation. The water shed segmentation compares each pixel with the other pixels to separate the tumour from the image. The watershed algorithm was proposed by F.Meyer. After successful segmentation of the image, the morphological operation is performed. The process of probing a small shape of the image by placing over all possible regions of the image is performed; so as to compare with the corresponding pixels of the GDI.

The watershed algorithm utilizes the gradient magnitude as the function for segmentation. The foreground objects are marked by the morphological techniques. The background pixels in dark are computed with thin boundaries in the image to obtain the brain tumour. The desired output is obtained by taking the watershed transform of the gradient magnitude.

\section{H. Image Extraction}

The image of the desired portion is extracted for the tumour region. The analysis of the tumour properties like weight, dimension, size, and numbers are performed for any desired patient.

\section{Results and Discussions}

The MRI is converted into the GDI image with the resolution of $2^{8}$ bits. Fig. 1 shows the image of the $2^{8}$ bit resolution of the human skull by pre-processing. Fig. 2 depicts the detection of the edges for the desired region of interest. That is, the edge of the human skull in the GDI image. The desired region of the tumour is found by the ROI of the image as shown in Fig.3. The noises in the digital pixels are eradicated by the filtering process. The enhancement of the tumour region in the human skull in presented in the Fig.4. In order to separate the tumour region from the image, the segmentation is performed. The segmented image using the water shed algorithm is given in Fig.5. The image extracted with the tumour region is shown in the Fig.6. The extraction of the tumour region of the image is evaluated with the score plot each in vertical and horizontal direction as given in the Fig.7 and 8 respectively.

\section{Conclusion}

The diagnosis of the tumour in the human brain is successfully determined by using the water shed segmentation method. The water shed algorithm utilized for the segmentation proves to be efficient and effective in the analysis of the tumour cells in the MRI based Image. Future work could be extended with the segmentation of the colour images by contouring technique and image features could be extracted in 3-Dimensional form for detailed analysis. 


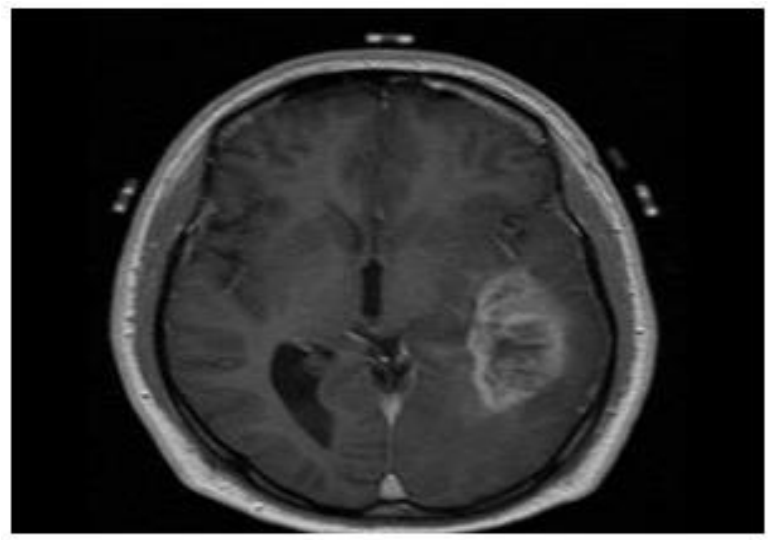

Fig. 1: GDI of the MRI with $2^{8}$ bits resolution

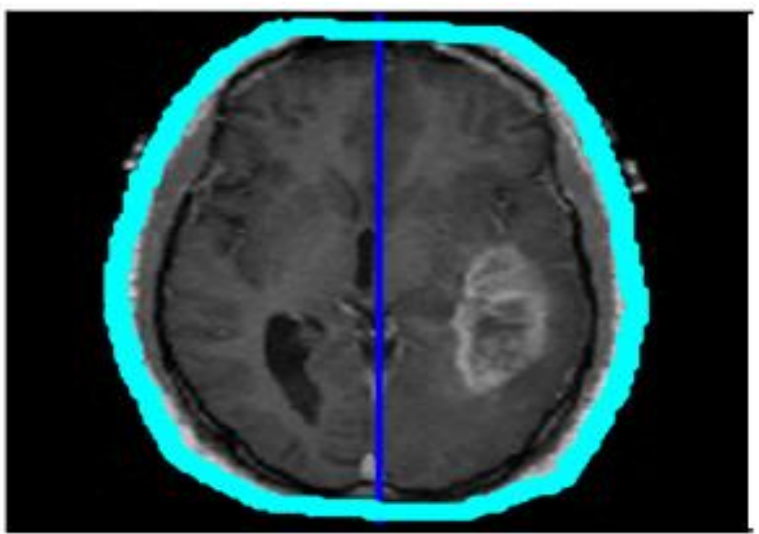

Fig. 2: Edge Detection of the skull

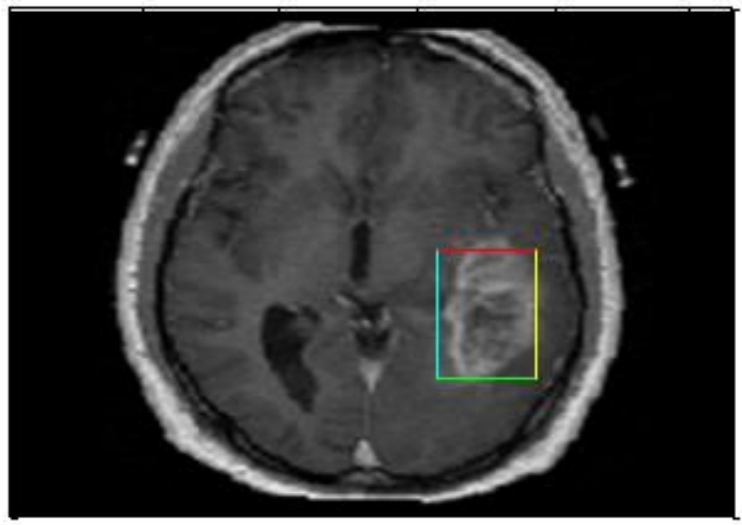

Fig. 3: Region of Identification of Tumour

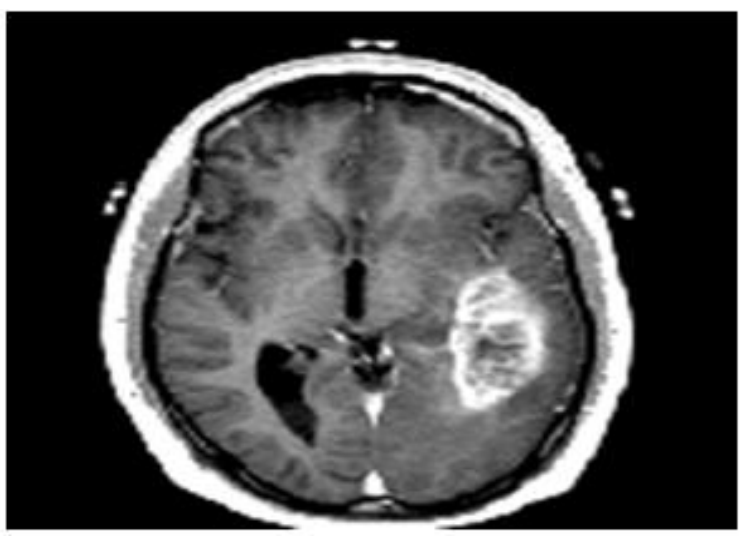

Fig. 4: Enhanced image of the ROI

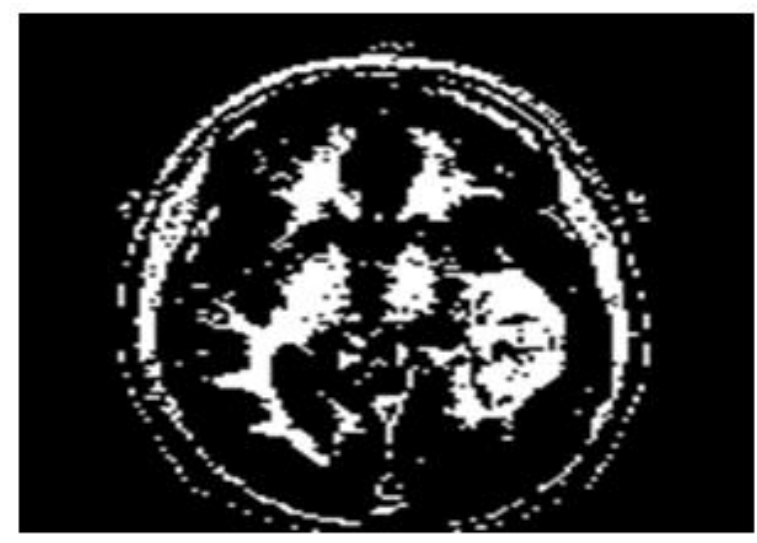

Fig. 5: Water shed segmentation of the Human tumour skull

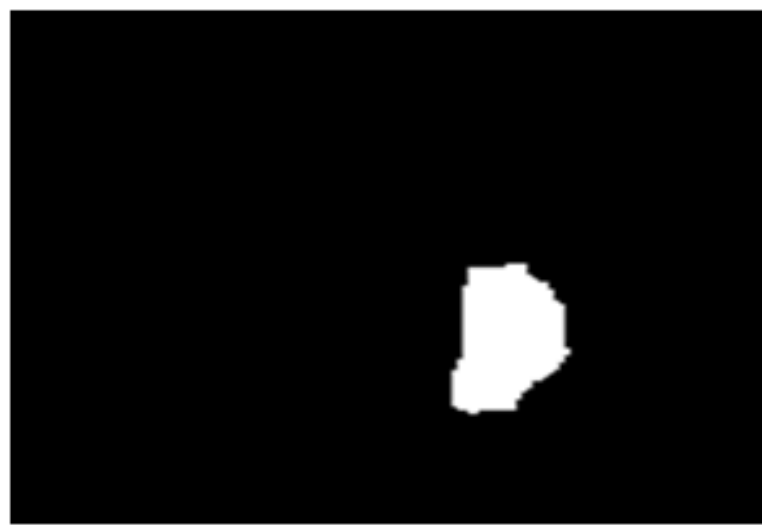

Fig. 6: Extracted image of the tumour

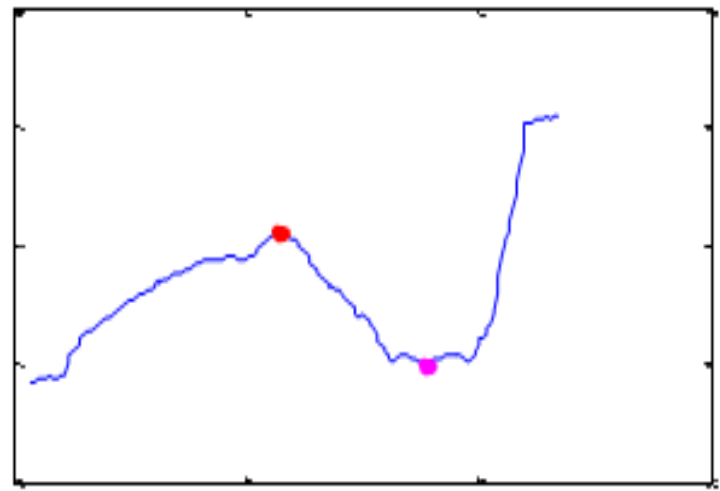

Fig. 7: Score plot for vertical direction

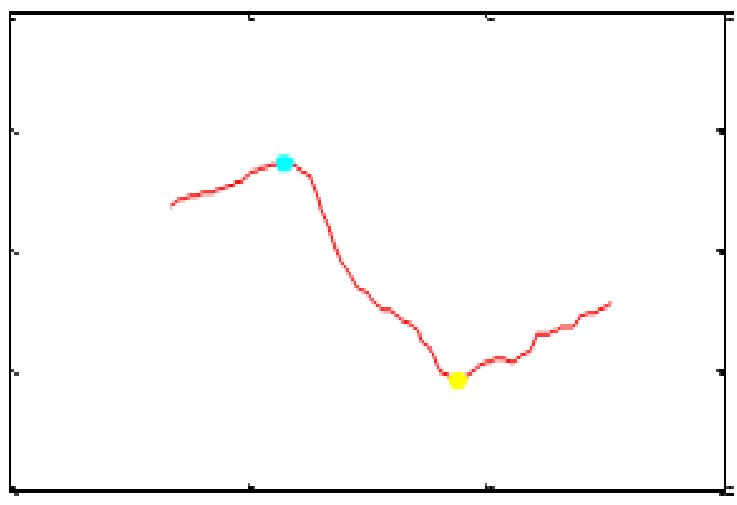

Fig. 8: Score plot for horizontal direction 


\section{Acknowledgement}

The Author would like to thank his Guide and College Management for the sustained encouragement in completing this work.

\section{References}

[1] Liu, M. Li, J. Wang, F. Wu, T. Liu, and Y. Pan, "A survey of MRI-based brain tumour segmentation methods," Tsinghua Science and Technology, Vol. 19(6), pp. 578-595, 2014

[2] Parmeet Kaur, Harish Kundra, "Usage of Brain Tumor Segmentation in MRI Images Using Intelligent Water Drops Algorithm" International Journal of Engineering And Computer Science ISSN:2319-7242,Vol 6(6), pp. 21732-21736, 2017.

[3] Qazi Nida-Ur-Rehman1, Imran Ahmed, Ghulam Masood, NajamU-Saquib, Muhammad Khan, Awais Adnan, "Segmentation of Brain Tumor in Multimodal MRI using Histogram Differencing \& KNN",International Journal of Advanced Computer Science and Applications, Vol. 8( 4), pp.249-256, 2017

[4] T.Kalaiselvi, S.Vijayalakshmi, K.Somasundara, "Segmentation Of Brain Portion From MRI Of Head Scans Using Kmeans Cluster", International Journal Of Computational Intelligence And Informatic ,Vol. 1,No. 1, Pp 75-79,2011.

[5] Rohini Paul Joseph , C. Senthil Singh , M.Manikandan3, "Brain Tumor MRI Image Segmentation And Detection In Image Processing",

[6] S. Damodharan and D. Raghavan, "Combining tissue segmentation and neural network for brain tumour detection," International Arab Journal of Information Technology, Vol. 12(1), pp. 42-52, 2015.

[7] Nilesh Bhaskarrao Bahadure, Arun Kumar Ray, and Har Pa Thethi, "Image Analysis for MRI Based Brain Tumor Detection and Feature Extraction Using Biologically Inspired BWT and SVM", International Journal of Biomedical Imaging, Hindawi Publications, pp: 1-12, 2017.

[8] A. Chaddad, "Automated feature extraction in brain tumour by magnetic resonance imaging using gaussian mixture models," International Journal of Biomedical Imaging, vol. 2015, Article ID 868031, 11 pages, 2015.

[9] D. Jagadish, K. Sindhu Priya, V. Saranya, A.S. Akshara, G. Mohanapriya, "Detection of Brain Tumour by Image Fusion Based on Discrete Wavelet Transform" Journal of Chemical and Pharmaceutical Sciences ISSN: 0974-2115 , Special Issue 6, pp:158-161, 2017.

[10] Abd El Kader Isselmou, Shuai Zhang, Guizhi Xu, "A Novel Approach for Brain Tumor Detection Using MRI Images", J. Biomedical Science and Engineering, Vol: 9, pp: 44-52, 2016.

[11] K.Bhima and A.Jagan, "Analysis of MRI based Brain Tumor Identification using Segmentation Technique", International Conference on Communication and Signal Processing, pp: 2109 2113, 2016.

[12] R. Kalaivani, K. Ramash Kumar, S. Jeevananthan, "Implementation of VSBSMC plus PDIC for Fundamental Positive Output Super Lift-Luo Converter," Journal of Electrical Engineering, Vol. 16, Edition: 4, 2016, pp. 243-258

[13] K. Ramash Kumar,'Implementation of Sliding Mode Controller plus Proportional Integral Controller for Negative Output Elementary Boost Converter," Alexandria Engineering Journal (Elsevier), 2016, Vol. 55, No. 2, pp. 1429-1445.

[14] P. Sivakumar, V. Rajasekaran, K. Ramash Kumar, "Investigation of Intelligent Controllers for Varibale Speeed PFC Buck-Boos Rectifier Fed BLDC Motor Drive," Journal of Electrical Engineering (Romania), Vol.17, No.4, 2017, pp. 459-471.

[15] K. Ramash Kumar, D.Kalyankumar, DR.V.Kirbakaran” An Hybrid Multi level Inverter Based DSTATCOM Control, Majlesi Journal of Electrical Engineering, Vol. 5. No. 2, pp. 17-22, June 2011, ISSN: 0000-0388.

[16] K. Ramash Kumar, S. Jeevananthan, "A Sliding Mode Control for Positive Output Elementary Luo Converter," Journal of Electrical Engineering, Volume 10/4, December 2010, pp. 115-127.

[17] K. Ramash Kumar, Dr.S. Jeevananthan," Design of a Hybrid Posicast Control for a DC-DC Boost Converter Operated in Continuous Conduction Mode" (IEEE-conference PROCEEDINGS OF ICETECT 2011), pp-240-248, 978-1-4244$7925-2 / 11$
[18] K. Ramash Kumar, Dr. S. Jeevananthan,” Design of Sliding Mode Control for Negative Output Elementary Super Lift Luo Converter Operated in Continuous Conduction Mode", (IEEE conference Proceeding of ICCCCT-2010), pp. 138-148, 978-1-4244-7768$5 / 10$

[19] K. Ramash Kumar, S. Jeevananthan, S. Ramamurthy" Improved Performance of the Positive Output Elementary Split InductorType Boost Converter using Sliding Mode Controller plus Fuzzy Logic Controller, WSEAS TRANSACTIONS on SYSTEMS and CONTROL, Volume 9, 2014, pp. 215-228.

[20] N. Arunkumar, T.S. Sivakumaran, K. Ramash Kumar, S. Saranya "Reduced Order Linear Quadratic Regulator plus Proportional Double Integral Based Controller for a Positive Output Elementary Super Lift Luo-Converter," JOURNAL OF THEORETICAL AND APPLIED INFORMATION TECHNOLOGY, July 2014. Vol. 65 No.3, pp. 890-901.

[21] Arunkumar, T.S. Sivakumaran, K. Ramash Kumar, "Improved Performance of Linear Quadratic Regulator plus Fuzzy Logic Controller for Positive Output Super Lift Luo-Converter," Journal of Electrical Engineering, Vol. 16, Edition:3, 2016, pp. 397-408.

[22] S.V.Manikanthan and K.Baskaran "Low Cost VLSI Design Implementation of Sorting Network for ACSFD in Wireless Sensor Network", CiiT International Journal of Programmable Device Circuits and Systems,Print: ISSN 0974 - 973X \& Online: ISSN 0974 - 9624, Issue : November 2011, PDCS112011008.

[23] T. Padmapriya and V. Saminadan, "Distributed Load Balancing for Multiuser Multi-class Traffic in MIMO LTE-Advanced Networks", Research Journal of Applied Sciences, Engineering and Technology (RJASET) - Maxwell Scientific Organization ISSN: 2040-7459; e-ISSN: 2040-7467, vol.12, no.8, pp:813-822, April 2016 